\title{
Implementation of numerical methods for the electromagnetic analysis of periodic media: Application in optical wavelengths and computational optimization
}

\section{Implementación de métodos numéricos para el análisis electromagnético de medios periódicos: aplicación en longitudes de onda ópticas y optimización computacional}

\author{
Jorge Francés $\left.{ }^{*}, S\right)$ \\ Departamento de Física, Ingeniería de Sistemas y Teoría de la Señal, e Instituto Universitario de Física \\ Aplicada a las Ciencias y las Tecnologías, Universidad de Alicante, Apartado 99, 03080 Alicante, Spain. \\ ${ }^{(*)}$ Email: jfmonllor@ua.es \\ S: miembro de SEDOPTICA / SEDOPTICA member \\ Received / Recibido: 24/10/2014. Revised / Revisado: 11/11/2014. Accepted / Aceptado: 12/11/2014.
}

DOI: http://dx.doi.org/10.7149/OPA.47.4.261

\begin{abstract}
:
This paper summarizes the contributions of a $\mathrm{PhD}$ focused on developing a set of numerical methods for the analysis of periodic diffractive optical devices such as holographic diffractive devices, thin film filters or diffraction gratings. For the rigorous and comprehensive analysis of these devices in optical wavelengths the finite difference time domain (DFDT) method was considered being compared to classical theories. Application of DFDT in optical wavelengths implies a considerable computational cost. Therefore, this method was accelerated for the most common computing platforms: CPU (Central Processing Unit) and GPU (Graphic Processing Unit).
\end{abstract}

Key words: Holography, Finite Differences, Optimization, High Performance Computing, Diffractive Optics.

\section{RESUMEN:}

En este artículo se hace una revisión de las aportaciones de la Tesis Doctoral en la que se ha desarrollado una serie de métodos numéricos para el análisis de dispositivos ópticos difractivos periódicos como pueden ser Redes de Difracción Holográficas (RDH), filtros de capa delgada o redes de apertura. Para el análisis riguroso y completo de estos medios en longitudes de onda ópticas se ha recurrido al método de las diferencias finitas en el dominio del tiempo (DFDT), siendo contrastado mediante teorías clásicas. La aplicación del método de las DFDT en longitudes de onda ópticas implica un coste computacional considerable. Por ello, este método se ha acelerado en las plataformas de cálculo más comunes en la actualidad: CPU (Central Processing Unit) y GPU (Graphic Processing Unit).

Palabras clave: Holography, Finite Differences, Optimization, High Performance Computing, Diffractive Optics.

\section{REFERENCES AND LINKS / REFERENCIAS Y ENLACES}

[1]. T. Cao, M. J. Cryan, Y.-L. D. Ho, I. J. Craddock, C. J. Railton, "Fast-light based pulse compression in 2-d photonic crystal waveguides", J. Lightwave Technol. 25, 2590-2598 (2007). DOI

[2]. S. Fan, P. R. Villeneuve, J. D. Joannopoulos, M. J. Khan, C. Manolatou, H. A Haus, "Theoretical analysis of channel drop tunneling processes", Phys. Rev. B 59, 15882-15892 (1999). DOI

[3]. Y. Shi, D. Dai, S He, "Proposal for an ultracompact polarization-beam splitter based on a photoniccrystal assisted multimode interference coupler", IEEE Photon. Technol. Lett. 19, 825-827 (2007). DOI 
[4]. O. Burmeister, M. Britzger, A Thüring, D. Friedrich, F. Brückner, K. Danzmann, R. Schnabel, "All reflective coupling of two optical cavities with 3-port diffraction gratings", Opt. Express 18, 91199132 (2010). DOI

[5]. T. Clausnitzer, E.-B. Kley, A. Tünnermann, A. Bunkowski, O. Burmeister, K. Danzmann, R. Schnabel, S. Gliech, A. Duparré, "Ultra low-loss low-efficiency diffraction gratings", Opt. Express 13, 4370-4378 (2005). DOI

[6]. T. W. Stone, Selected Papers on Holographic and Diffractive Lenses and Mirrors, SPIE Milestone Series, volume MS 34 (1991).

[7] A. K. Richter, F. P. Carlson, "Holographically generated lens”, Appl. Opt. 13, 2924-2930 (1974). DOI

[8]. S. Tibuleac, D. Wawro, R. Magnuson, "Resonant diffractive structures integrating waveguide-gratings on optical fiber endfaces", LEOS '99, IEEE Lasers and Electro-Optics Society, $12^{\text {th }}$ Annual Meeting, Vol. 2, p. 874 (1999).

[9] M. A. Hembrecht, "Optical coatings for mems devices", 2010 Conference on Lasers and Electro-Optics (CLEO) and Quantum Electronics and Laser Science Conference (QELS), Vol. 1 (2010).

[10]. D. B. Kirk, W-M.W. Hwu, Programming Massively Parallel Processsors: A Hands-on Approach, Morgan Kaufmann (2010).

[11]. F. Shen, A. Wang, "Fast-Fourier-transform based numerical integration method for the RayleighSommerfeld diffraction formula", Appl. Opt. 45, 1102-1110 (2006). DOI

[12]. G. N. Lawrence, S.-H. Hwang, "Beam propagation in gradient refractive-index media", Appl. Opt. 31, 5201-5210 (1992). DOI

[13]. A. D. Papadopoulos, E. N. Glytsis, "Finite-difference-time-domain analysis of finite-number-of-periods holographic and surface-relief gratings", Appl. Opt. 47, 1981-1994 (2008). DOI

[14]. C. M. Dissanayake, M. Premaratne, I. D. Rukhlenko, G. P. Agrawal, "FDTD modeling of anisotropic nonlinear optical phenomena in silicon waveguides", Opt. Express 18, 21427-21448 (2010). DOI

[15] D. M. H. Leung, B. M. A. Rahman, K. T. V. Grattan, "Numerical analysis of asymmetric silicon nanowire waveguide as compact polarization rotator", IEEE Photonics J. 3, 381-389 (2011). DOI

[16] J. Francés, Implementación de Métodos Numéricos para el Análisis Electromagnético de Medios Periódicos: Aplicación en Longitudes de Onda Ópticas y Optimización Computacional. PhD Thesis, Universidad de Alicante, Spain (2011).

[17]. J. Francés, C. Neipp, M. Pérez-Molina, M. L. Álvarez, A. Beléndez, "Aplicación del algoritmo de las diferencias finitas en el dominio del tiempo en redes de difracción holográficas", Opt. Pura Apl. 42, 139-145 (2009).

[18]. J. Francés, M. Pérez-Molina, C. Neipp, A. Beléndez, "Rigorous interference and diffraction analysis of diffractive optic elements using the finite-difference time-domain method", Comput. Phys. Commun. 181, 1963-1973 (2010). DOI

[19]. J. Francés, C. Neipp, A. Márquez, A. Beléndez, I. Pascual, "Analysis of reflection gratings by means of a matrix method approach", Prog. Electromagnetic Res. 118, 167-183 (2011). DOI

[20]. J. Francés, S. Bleda, C. Neipp, A. Márquez, I. Pascual, A. Beléndez, "Performance analysis of the FDTD method applied to holographic volume gratings: Multi-core CPU versus GPU computing", Comput. Phys. Commun. 184, 469-479 (2013). DOI

[21]. M. G. Moharam, T. K. Gaylord, "Rigurous coupled-wave analysis of planar-grating diffraction", J. Opt. Soc. Am. 71, 811-818 (1981). DOI

[22]. M. G. Moharam, T. K. Gaylord, "Coupled-wave analysis of reflection gratings", Appl. Opt. 20, 240-244 (1981). DOI

[23]. M. G. Moharam, T. K. Gaylord, "Rigorous coupled-wave analysis of metallic surface-relief gratings", J. Opt. Soc. Am. A. 3, 1780-1787 (1986). DOI

[24]. L. Carretero, M. Pérez-Molina, P. Acebal, S. Blaya, A. Fimia, "Matrix method for the study of wave propagation in one-dimensional general media", Opt. Express 14, 11385-11391 (2006). DOI

[25]. J. Lekner, Theory of Reflection of Electromagnetic and Particle Waves, Kluwer Academic Publishers Group (1987). 
[26]. G. Blanch, Handbook of Mathematical Functions with Formulas, Graphs, and Mathematical Tables, $\mathrm{Ch}$. 20, pp. 722-748, Dover Publications (1972).

[27]. D. M. Sullivan, Electromagnetic Simulation using the FDTD Method, IEEE Press Editorial Board (2000). DOI

[28]. C. A. Balanis, Advanced Engineering Electromagnetics, Wiley (1989).

[29]. J. Francés, M. Pérez-Molina, S. Bleda, E. Fernández, C. Neipp, A. Beléndez, "Educational software for interference and optical diffraction analysis in Fresnel and Fraunhofer regions based on MATLAB GUIs and the FDTD method", IEEE T. Educ. 55, 118-125 (2012). DOI

[30].R. R. Boye, R. W. Ziolkowski, R. K. Kostuk, "Resonant waveguide-grating switching device with nonlinear optical material", Appl. Opt. 38, 5181-5185 (1999). DOI

[31].E. Popov, M. Nevierè, B. Gralak, G. Tayeb, "Staircase approximation validity for arbitrary-shaped gratings", J. Opt. Soc. Am. A 19, 32-42 (2002). DOI

[32]. K-H. Kim, K. Kim, Q-H. Park, "Performance analysis and optimization of three-dimensional FDTD on GPU using roofline model”, Comput. Phys. Commun. 182, 1201-1207 (2011). DOI

\section{Introducción}

En la última década, la óptica ha experimentado una importante revolución debido a la era de la información en la que está inmersa la sociedad. La mejora de las comunicaciones, el aumento del flujo de datos en sistemas de telecomunicaciones y las mejoras en las técnicas de imagen son algunas de las facetas que se han visto beneficiadas a raíz de los avances científicos en el campo de la Óptica. Por ello, los investigadores centran esfuerzos en el desarrollo y mejora de herramientas que permitan el estudio riguroso de dispositivos ópticos. Un grupo muy significativo de los dispositivos ópticos difractivos más utilizados son aquellos que presentan variaciones de las características físicas del medio de tipo periódico. La propagación del campo electromagnético en el interior de estos medios presenta un conjunto de características cuyas aplicaciones son muy diversas. Algunos de estos fenómenos son la reflexión de Bragg y la presencia de bandas fotónicas prohibidas, donde éstas últimas se traducen en un rango espectral en el que la propagación de los fotones es de tipo evanescente. La aplicación de estas características ha permitido el diseño de multitud de dispositivos ópticos como pueden ser guías de onda planas [1], filtros fotónicos de canal [2], acopladores [3], redes de difracción [4,5], elementos ópticos holográficos [6], lentes [7], sistemas de interconexión óptica holográfica [8], computación óptica [9], etcétera.
Múltiples dispositivos fotónicos funcionan en base a cuestiones ligadas a la teoría de la difracción. Dentro de la óptica difractiva destacan por sus múltiples propiedades y aplicaciones, las redes de difracción. De forma general, una red de difracción se constituye mediante una serie de elementos difractores (aperturas u obstáculos), los cuales producen alteraciones periódicas en la fase y/o en la amplitud. El caso más sencillo de este tipo de dispositivos son las conocidas redes de difracción de aperturas, cuya aplicación más popular es la que se conoce como el experimento de Young de la doble rendija, realizado por Thomas Young en 1801. Existe otro tipo de redes de difracción basadas en la variación del índice de refracción del medio. Uno de los casos particulares de este tipo de dispositivos son las redes de difracción obtenidas mediante técnicas holográficas.

Con el objetivo de analizar y predecir el comportamiento de la luz en dispositivos ópticos, numerosos investigadores han dedicado sus esfuerzos a la disciplina de la simulación numérica en el campo de la óptica difractiva [1113] y la fotónica [14,15]. En este trabajo se estudian diferentes métodos analíticos y numéricos y su aplicación a elementos ópticos difractivos en longitudes de onda ópticas. Concretamente, se ha recurrido al método numérico de las DFDT. El método se basa en realizar una discretización del medio para calcular de forma numérica las componentes del campo electromagnético en función del tiempo y 
del espacio. Sin embargo, uno de los inconvenientes principales en el uso del método de las DFDT en longitudes de onda ópticas es la necesidad de reducción de las resoluciones espaciales y temporales, para asegurar tanto estabilidad como convergencia en el método. Por ello, la simulación de grandes regiones comparadas con la longitud de onda de trabajo (escenario común en óptica difractiva) y un elevado número de pasos temporales aumenta el coste computacional en términos de uso de memoria y tiempo de simulación. No obstante, en las últimas décadas, los microprocesadores han permitido reducir los tiempos de simulación. El aumento en la velocidad de estos procesadores ha revolucionado el mundo de la informática en pocos años, mejorando los interfaces de usuario, y ampliando las funcionalidades del software. Por otro lado, en los últimos años las GPU están siendo utilizadas de forma masiva en aplicaciones relacionadas con la investigación debido a su elevado potencial de cálculo. En la Fig. 1 se muestra la evolución temporal desde el 2003 del rendimiento de los GPU de NVIDIA comparada con la evolución de los procesadores Intel.

En este trabajo se resumen los desarrollos y resultados derivados en [16], los cuales principalmente se pueden agrupar en cuatro secciones: una primera toma de contacto con el método numérico y su aplicación y posterior discusión a dispositivos ópticos difractivos de carácter periódico $[17,18]$; en segundo lugar se muestran resultados derivados de la validación teórica del método DFDT en forma de aplicación docente interactiva; en tercer lugar se recogen diferentes formalismos teóricos analíticos para la comparativa del método numérico aplicado a $\mathrm{RDH}$ [19] y finalmente se detallan las estrategias

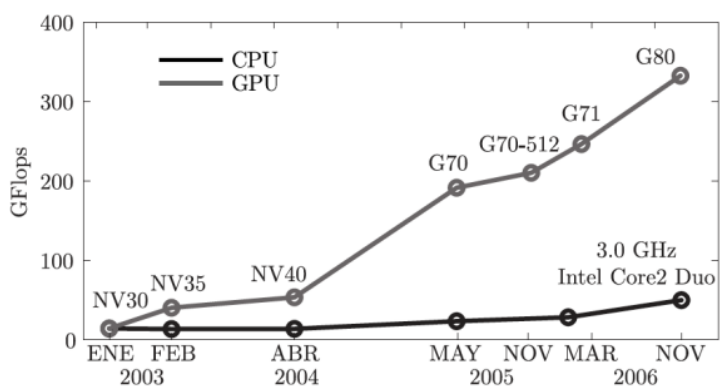

Fig. 1. Evolución temporal del rendimiento de los GPU y CPU [10]. de optimización consideradas tanto en CPU como GPU para reducir los costes de cálculo del método DFDT [20]. Por ello, las bases teóricas que engloban estas acciones se detallas en la sección 2. Posteriormente se recogerán algunos de los resultados más significativos de las líneas anteriormente enumeradas en la sección 3 y finalmente se darán las conclusiones derivadas de este trabajo.

\section{Herramientas y métodos}

En esta sección se detallarán las herramientas utilizadas para el análisis teórico de los elementos ópticos difractivos estudiados en este trabajo.

\subsection{Teoría rigurosa de ondas acopladas}

La TROA analiza la difracción producida por una onda plana electromagnética incidente a una red de difracción con ángulo arbitrario de incidencia y que está situada entre el medio I y el medio III, tal y como se ilustra en la Fig. 2.

Tal y como se aprecia en la Fig. 2, el problema bajo estudio se basa en el análisis de una onda plana en incidencia oblicua con polarización TE, sobre un medio dieléctrico periódico y para este caso, sin pérdidas. La variación del índice de permitividad dieléctrica relativa en el medio II puede formularse de manera sencilla de la siguiente forma [21-23]:

$$
\epsilon_{I I}(x, z)=\epsilon_{r 0}+\Delta \epsilon[K(x \sin \phi+z \cos \phi)] .
$$

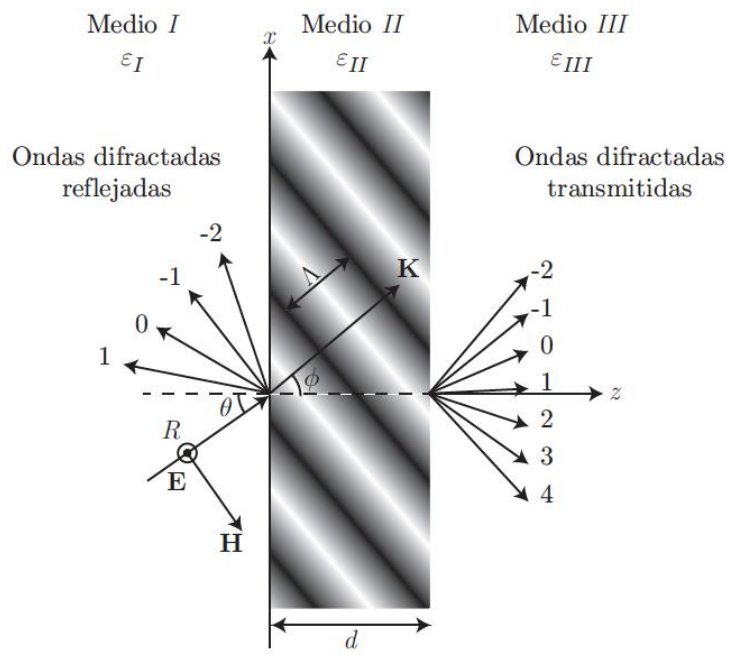

Fig. 2. Geometría de una red de difracción holográfica que contempla los diferentes órdenes difractados y transmitidos [21] 
Los valores de la constante dieléctrica en los medios I y III toman los valores de $\epsilon_{I}$ y $\epsilon_{I I I}$ respectivamente. El problema reside en encontrar la solución de la ecuación de onda en cada una de las tres regiones para aplicar las condiciones de contorno relacionadas con la continuidad del campo eléctrico y magnético tangencial en las dos interfases $(z=0$ y $z=d$ ) que permitan resolver el sistema de ecuaciones planteado:

$$
\mathbf{S}^{\prime \prime}=\left[\mathbf{K}_{x}^{2}-\widehat{\mathbf{K}}\right] \mathbf{S},
$$

donde $\mathbf{S}^{\prime \prime}$ y $\mathbf{S}$ son vectores de $2 N+1$ filas y representan las amplitudes normalizadas de los diferentes modos. La prima denota la derivada respecto la coordenada $z$. La matriz $\mathbf{K}_{x}$ es una matriz diagonal con los valores de $k_{x n}$ y la matriz $\widehat{\mathbf{K}}$ son las componentes de Fourier del medio y que se sitúan en la diagonal y adyacentes a la misma. Para el caso de redes de difracción con patrón sinusoidal, únicamente tendríamos dos coeficientes alrededor de la diagonal y el término de continua, mientras que para perfiles más complejos, se podrían tomar hasta $p$ coeficientes.

\subsection{Método matricial con las funciones de Mathieu}

El análisis teórico de redes de difracción no inclinadas necesita de formalismos añadidos a los vistos hasta el momento debido a que la TROA es válida para cualquier ángulo de incidencia $\theta$ y para ángulos de inclinación de las franjas de interferencia distinto a $180^{\circ}$ (vector de red paralelo a la normal de la red). Para este caso particular, las franjas de interferencia son paralelas al plano de incidencia, constituyendo lo que se conoce como una red de difracción holográfica de reflexión pura y simétrica. En este tipo de configuraciones, la modulación no es periódica de manera rigurosa ya que en la red hay número finito de períodos, por lo que la TROAK en su forma estándar no es aplicable. Por ello, es necesario recurrir a otros formalismos que modelicen de una manera más precisa el comportamiento de este tipo de dispositivos.

En este trabajo se extiende el formalismo expuesto en [24], donde se presenta un método matricial para evaluar la transmitancia para el caso unidimensional de una red de difracción en reflexión en incidencia normal. Este tipo de métodos se han utilizado con anterioridad en medios estratificados y básicamente la idea se basa en definir una matriz que caracterice el medio y que relacione las componentes del campo magnético de ambos extremos de la capa (matriz de capa M). La adición de capas puede modelizarse por una simple multiplicación de matrices elementales. En nuestro caso particular, el método se aplica al análisis del campo electromagnético en $\mathrm{RDH}$ no inclinadas para cualquier ángulo de incidencia. Para ello, se calcula la matriz que caracteriza este tipo de redes a partir de dos soluciones particulares de la ecuación de Helmholtz [25].

En el interior del medio periódico, $E(z)$ verifica la siguiente ecuación diferencial de segundo orden:

$$
\frac{d^{2} E}{d z^{2}}+\left(k_{0}^{2} \epsilon(z)-k_{x}^{2}\right) E=0 .
$$

Para obtener el conjunto de dos soluciones linealmente independientes de la Ec. (3), se puede deducir de manera sencilla que a partir de transformaciones elementales puede obtenerse la ecuación de Mathieu [26]:

$$
\frac{d^{2} E}{d u^{2}}+(a-2 q \cos (2 u)) E=0,
$$

teniendo en cuenta los siguientes cambios de variable:

$$
\begin{gathered}
u=\frac{K z}{2}, \\
a=\frac{4}{K^{2}}\left(k_{0}^{2} \epsilon_{r 0}-k_{x}^{2}\right), \\
q=-\frac{2}{K^{2}} k_{0}^{2} \epsilon_{r 1} .
\end{gathered}
$$

Las dos soluciones linealmente independientes de la Ec. (4) están definidas por las funciones coseno y seno de Mathieu, como $C_{m}(a, q, u)$ y $S_{m}(a, q, u)$ respectivamente. En la Fig. 3 se representan ambas funciones en función del parámetro $u$ y para $q=1$.

$$
\begin{aligned}
& C_{m}(a, q, 0)=1 ; \frac{d}{d z} C_{m}(a, q, 0)=0, \\
& S_{m}(a, q, 0)=0 ; \frac{d}{d z} S_{m}(a, q, 0)=1 .
\end{aligned}
$$

Por lo tanto cualquier solución de la Ec. (3) puede expresarse en términos de las funciones de Mathieu: 
(a)

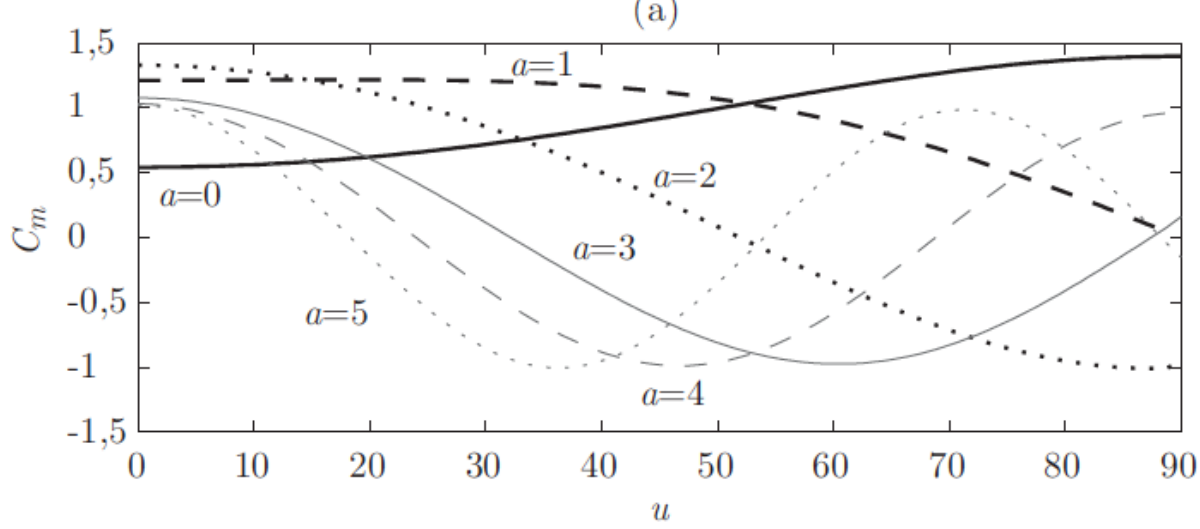

(b)

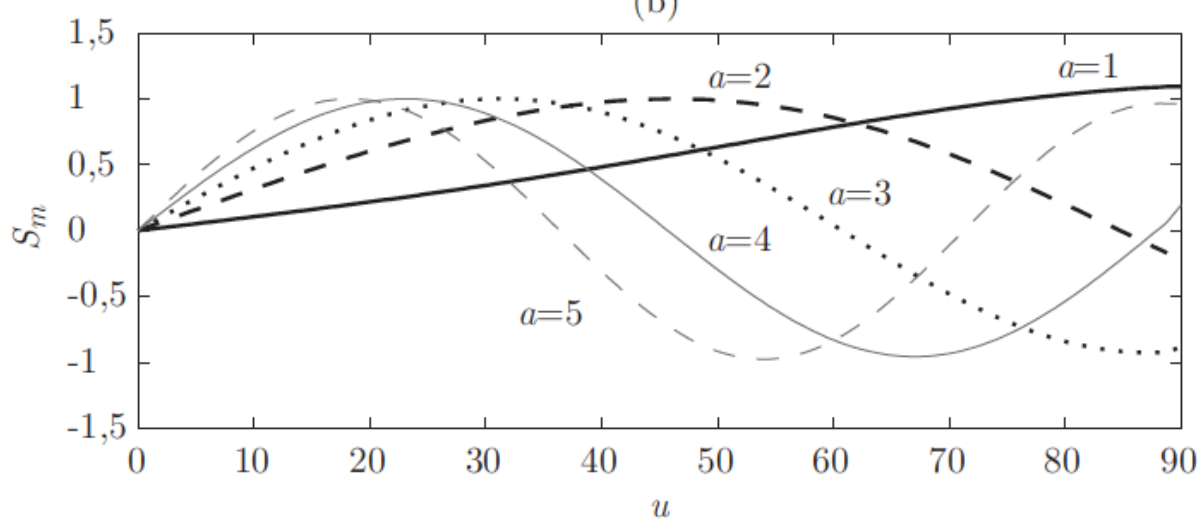

Fig. 3. Funciones de Mathieu $C_{m}$ y $S_{m}$ para $q=1$, en función del parámetro a definido por la Ec. (8). (a) Funciones par. (b) Funciones impar.

$E(z)=A C_{m}(a, q, K z / 2)+B S_{m}(a, q, K z / 2)$,

donde $A$ y $B$ son constantes arbitrarias. Llegado a esto punto se puede redefinir la matriz de capa M para un único período [25] en términos de:

$$
\begin{aligned}
& E_{c 1}=C_{m}(a, q, K z / 2), \\
& E_{c 2}=S_{m}(a, q, K z / 2) .
\end{aligned}
$$

Para el caso particular de una red de difracción en reflexión de espesor $d$, se pueden identificar expresiones analíticas sencillas para el cálculo de la reflectancia y transmitancia. El lector interesado puede recurrir a [19].

\subsection{Método DFDT}

El método DFDT se basa en resolver de forma numérica las ecuaciones de Maxwell en su forma diferencial $[27,28]$ :

$$
\begin{gathered}
\frac{\partial \mathbf{D}}{\partial t}=c_{0}(\nabla \times \mathbf{H}), \\
\mathbf{D}(\omega)=\epsilon_{r}(\omega) \cdot \mathbf{E}, \\
\frac{\partial \mathbf{H}}{\partial t}=-c_{0} \nabla \times \mathbf{E},
\end{gathered}
$$

donde $c_{0}$ es la velocidad de propagación de las ondas electromagnéticas en el vacío. El vector desplazamiento eléctrico está representado por D y los campos eléctricos están normalizados respecto a la impedancia intrínseca del vacío. Desarrollando estas expresiones mediante el método DFDT se pueden obtener un conjunto de expresiones en diferencias similares a la siguiente ecuación:

$$
\begin{gathered}
\left.D_{z}\right|_{i, j, k+\frac{1}{2}} ^{n+\frac{1}{2}}=\left.D_{z}\right|_{i, j, k+\frac{1}{2}} ^{n-\frac{1}{2}}+\frac{\Delta t}{\Delta x \sqrt{\mu_{0} \epsilon_{0}}} \times \\
\left(\left.H_{y}\right|_{i+\frac{1}{2}, j, k+\frac{1}{2}} ^{n}-\left.H_{y}\right|_{i-\frac{1}{2}, j, k+\frac{1}{2}} ^{n}-\right. \\
\left.\left.H_{x}\right|_{i, j+\frac{1}{2}, k+\frac{1}{2}} ^{n}+\left.H_{x}\right|_{i, j-\frac{1}{2}, k+\frac{1}{2}} ^{n}\right) .
\end{gathered}
$$

El resto de componentes en las Ecs. (12)-(14) se pueden derivar de forma similar a (15) $[17,18,20]$. La aplicación de estas expresiones es posible debido a la discretización espacial y temporal de las componentes del campo electromagnético, tal y como se aprecia en la Fig. 4. 


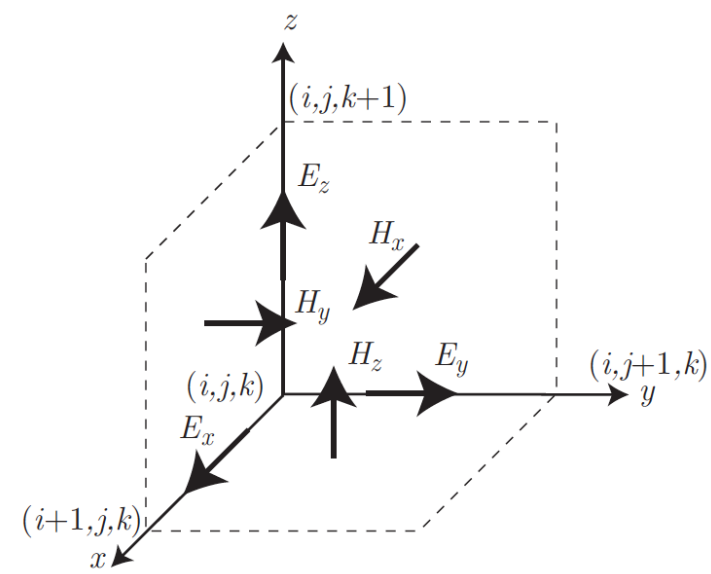

Fig. 4. Celda de Yee para el caso tridimensional.

\subsection{Optimización computacional del método DFDT}

La aplicación del método DFDT en longitudes de onda ópticas requiere de un elevado coste computacional en muchos casos. Para ello, se ha realizado una implementación optimizada tanto en microprocesadores convencionales como en las GPU NVIDIA, disponibles también en numerosas computadoras de propósito general. La aceleración de este método es necesaria para la aplicación del método a problemas más complejos como los relacionados con la formación de imágenes holográficas, almacenamiento de información o diseño y modelizado de lentes difractivas.

Las estrategias consideradas para la aceleración basada en microprocesadores está basada en el uso exhaustivo de instrucciones vectoriales y de la explotación de los recursos de los diferentes núcleos presentes en las CPU actuales, los cuales no son explotados por usuarios con conocimientos estándar de programación. Un ejemplo del cambio de paradigma en la programación con este tipo de estrategias se muestra en la Fig. 5. En la Fig. 5(a) se muestra cómo al utilizar instrucciones vectoriales, se puede reducir de forma significativa el tiempo de cómputo al operar de forma vectorial. Si a este hecho se le añade el factor de paralelismo disponible en la actualidad con los numerosos núcleos disponibles en los microprocesadores, se abra una puerta a la reducción de tiempos de ejecución mediante la (a)

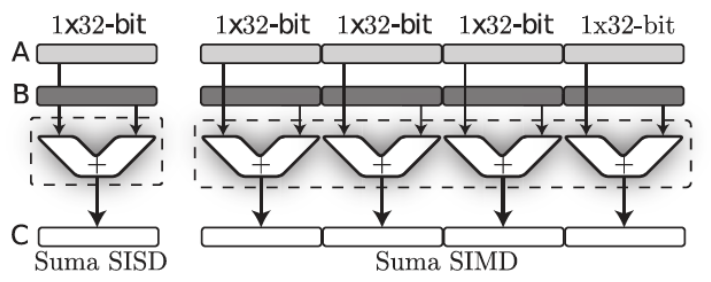

(c)

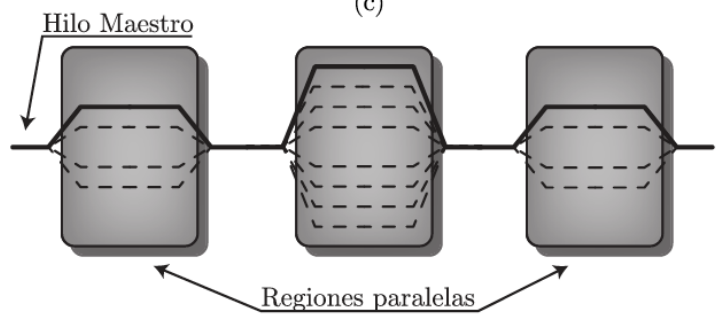

Fig. 5. (a) Ejemplo de una operación convencional en microprocesador. (b) Vectorización de instrucciones aritméticas mediante instrucciones SSE en microprocesadores. (c) Paralelización de las operaciones a lo largo de diferentes hilos en un mismo microprocesador con múltiples núcleos.

distribución de la carga de cálculo entre diferentes unidades de proceso (véase Fig. 5(b)).

Para el caso específico de la programación de métodos numéricos en GPU, es necesario tener en cuenta el elevado número de núcleos disponible en las GPU (del orden de 1000 en muchos casos), por lo que es necesario distribuir de forma eficiente la carga a través de dichas unidades de cálculo. En este trabajo, se ha adaptado el método DFDT a las GPU NVIDIA a través de CUDA (Computer Unified Device Architecture), que engloba tanto la parte hardware como la software, permitiendo al usuario utilizar lenguajes de alto nivel como $\mathrm{C}++$ o Fortran.

Un programa en CUDA se basa en la llamada a un kernel. Un kernel se ejecuta de forma paralela a lo largo de múltiples hilos. El programador y el compilador organizan estos hilos en bloques y su vez, la agrupación de bloques recibe el nombre de malla o grid. Cada hilo perteneciente a un bloque ejecuta una instancia del kernel y presenta un identificador que lo define en el bloque de forma inequívoca, este índice también permite seleccionar que datos o regiones de la malla DFDT va a ser actualizada por dicho hilo. Un bloque de hilos es un conjunto de hilos que trabajan de forma 


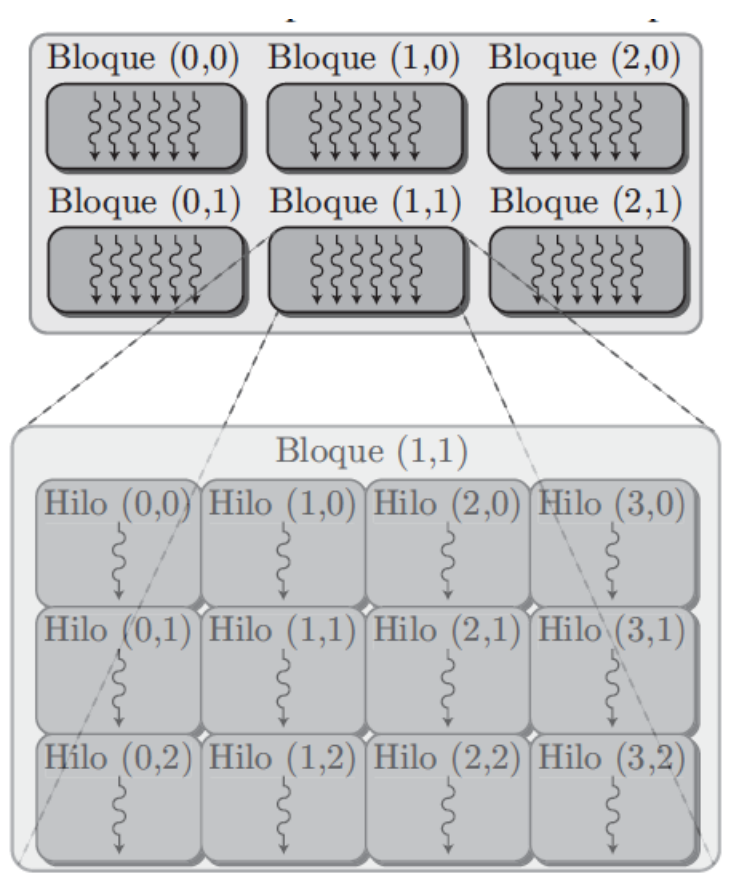

Fig. 6. Organización de los hilos en CUDA.

concurrente y que pueden colaborar entre ellos a través de barreras de sincronización y memoria de tipo compartida. En la Fig. 6 se ilustra la jerarquía de hilos, bloques y malla en CUDA. En el trabajo [20] se detallan las vicisitudes sobre la optimización realizada en ambas plataformas: CPU y GPU.

\section{Resultados}

El desarrollo de métodos numéricos para el análisis electromagnético ha permitido obtener diversos resultados de muy diferente índole. En primer lugar se resumen los resultados mostrados en los trabajos [16,29], los cuales son un ejemplo de la validación del método numérico implementado mediante el análisis de sistemas difractivos bien conocidos. En concreto se muestran los resultados del análisis de un problema canónico como puede ser el problema de la doble rendija de Young. El análisis se lleva a cabo analizando el patrón de difracción dos aperturas $(N=2)$ en función de la distancia de observación $L$. Las aperturas presentan un grosor $b$ de 40 celdas, y la separación entre ellas es $\mathrm{a}=80$ celdas. El tamaño de la celda en este caso se corresponde con una décima parte de la longitud de onda, por lo que $\Delta=\lambda / 10$, siendo $\lambda=633 \mathrm{~nm}$.

En la publicación [29] se recoge un mayor número de situaciones y comparativas, donde se enfatiza la posibilidad de visualizar en función del tiempo la distribución del campo eléctrico y por tanto del patrón de interferencia tanto en campo próximo, cercano y lejano. Mediante la aplicación interactiva desarrollada en [29], el usuario (que en este caso serían estudiantes de primer año de titulaciones de carácter físico e ingenieril) a la vez que observa cómo el campo electromagnético interactúa con las aperturas (fenómeno difícil de apreciar en un laboratorio), es también capaz de comparar y estudiar el rango de aplicabilidad de expresiones teóricas analíticas dadas para el análisis de la difracción en la región de Fresnel y Fraunhofer. Es interesante de cara a la docencia, que el estudiante desarrolle la capacidad de discernir el rango de validez y aplicabilidad de las expresiones teóricas proporcionadas en teoría. En los trabajos [16,29] se resumen dichas experiencias y se presentan un elevado número de resultados y situaciones similares a las mostradas en la Fig. 7. En concreto, en la Fig. 7 se muestran las curvas obtenidas con el método de las DFDT en su versión bidimensional junto con las curvas teóricas en la región de Fresnel y de Fraunhofer. La Fig. 7(a) muestra la irradiancia normalizada en función de el eje perpendicular a la apertura para una distancia de 300 celdas, mientras que la Fig. 7 (b) representa el patrón de interferencia a una distancia de 4500 celdas, lo cual se corresponde con la región de campo lejano.

Con este análisis se pretende analizar la precisión del método numérico tanto en la región de Fraunhofer como en la región de Fresnel y por lo tanto también en campo próximo. Tal y como se puede apreciar, el método numérico DFDT se comporta de forma precisa en ambos casos, mientras que la aproximación de Fraunhofer, claramente se muestra inexacta en las regiones de campo próximo.

La ventaja de utilizar una herramienta como DFDT es la posibilidad de estudiar de una forma robusta todos los fenómenos tanto en campo 

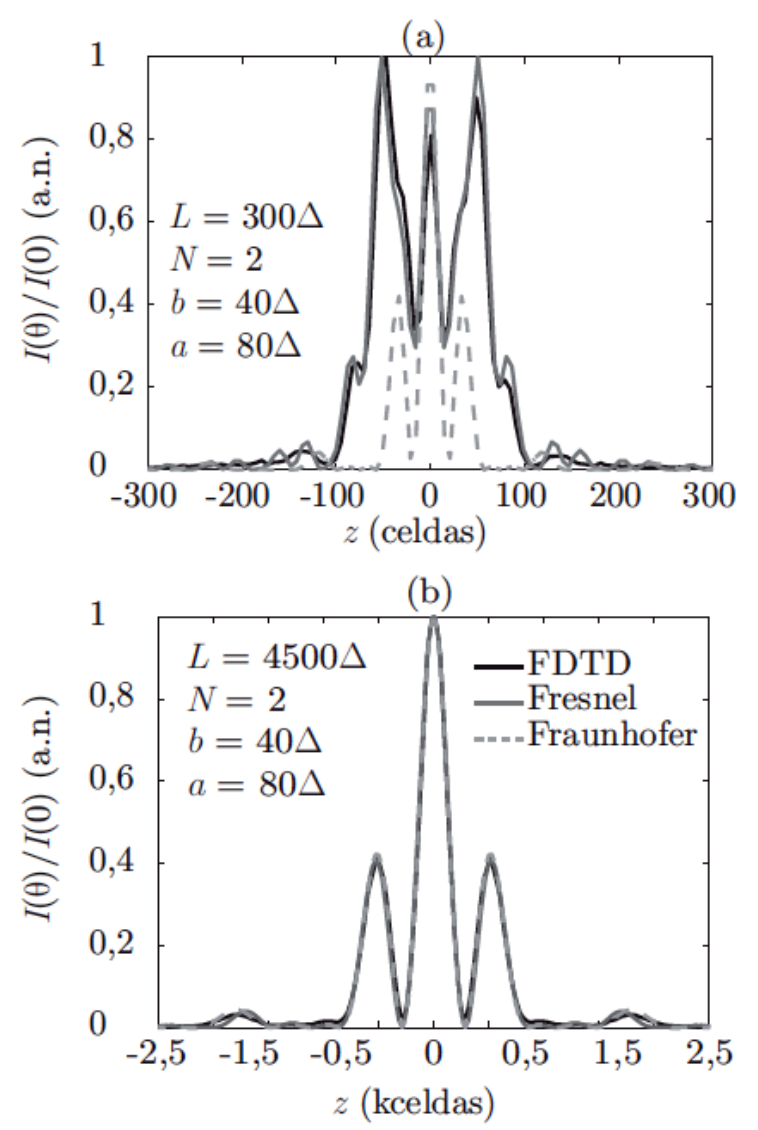

Fig. 7. Irradiancias normalizadas obtenidas por las expresiones teóricas en la región de Fresnel y Fraunhofer y numéricas a partir del método de las DFDT. Se ha estudiado el caso de dos aperturas para diferentes distancias entre el plano de aperturas y el plano de observación.

próximo y lejano con una única simulación. Además, el método DFDT permite obtener instantáneas del campo electromagnético en función del tiempo, facilitando el cálculo de parámetros indirectos con facilidad. Este análisis se muestra en $[16,17]$, donde se recogen las distribuciones del campo eléctrico para diferentes redes de difracción en diferentes situaciones de eficiencia. Ante esta situación se puede apreciar como el campo a la salida de la red, debida a la mezcla de órdenes, conforma una superposición de ondas planas con diferente vector de onda. Para ejemplificar este caso, en la Fig. 8 se representa el módulo del campo eléctrico $\left(\left|E_{z}\right|\right)$ para una red de difracción de transmisión similar a la mostrada en la secuencia de la Fig. 2, sin embargo en este caso se han aumentado las dimensiones de la malla a simular para identificar mejor los órdenes transmitido y difractado. Esta representación es

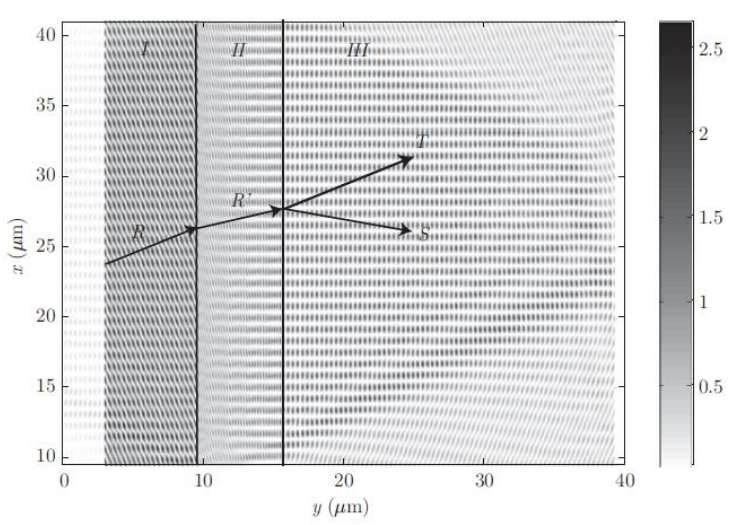

Fig. 8. Distribución del campo final para una red de difracción holográfica en transmisión, donde se visualiza la zona central de interferencia entre el haz transmitido y el difractado $[16,17]$.

un ejemplo cualitativo de la distribución del campo eléctrico en función de las regiones I, II y III, para este tipo de dispositivos, y del potencial del método DFDT para ser aplicado a situaciones complejas como la formación de lentes, formación de imágenes, almacenamiento de información holográfica, etcétera.

En primera instancia, el discernir las amplitudes del orden difractado y transmitido requeriría simulaciones de elevadas dimensiones para poder ver de manera aislada, cada uno de los órdenes a la salida de la red (tal y como se ha realizado para obtener el campo en la Fig. 8). Sin embargo, normalmente se suele calcular de forma numérica la distribución de campo lejano para obtener la amplitud del orden difractado, evitando aumentar el tamaño de simulación y en consecuencia el coste temporal del método. En puntos lo suficientemente alejados al plano de salida de red, es fácil identificar el orden transmitido y difractado de manera sencilla ya que no presentan solapamiento espacial. Por otro lado, el método DFDT requiere de una discretización espacial del medio, por lo que es necesario tomar ciertas consideraciones en los bordes de la malla en contacto con la red de difracción. Para ello, se han considerado perfiles con gradiente para encaminar la luz que se genera en dichas regiones al exterior de la malla, la cual presenta un contorno absorbente que reduce los posibles artefactos que se puedan generar y viajar de nuevo al centro del mallado. Este tipo de 


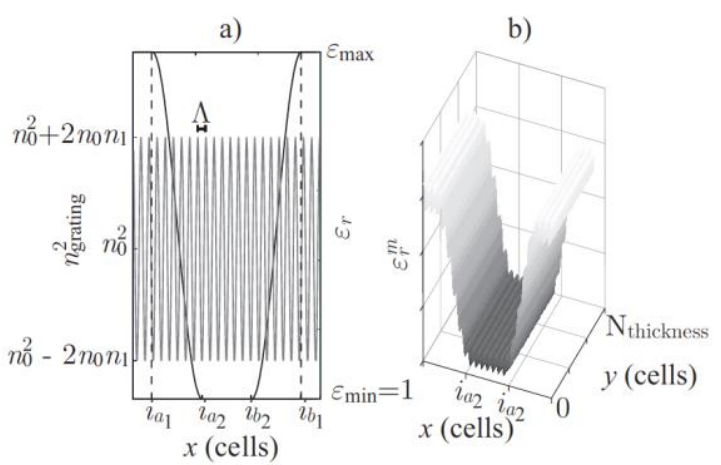

Fig. 9. Permitividad eléctrica e índice de refracción presente en una red de difracción en función de las variables espaciales. (a) Perfil del índice de refracción diseñado para encaminar la luz hacia los extremos absorbentes superpuesto con la variación sinusoidal del índice de refracción de la red (Francés et al. [16,18]). (b) Superposición de las contribuciones en (a) representando la permitividad eléctrica.
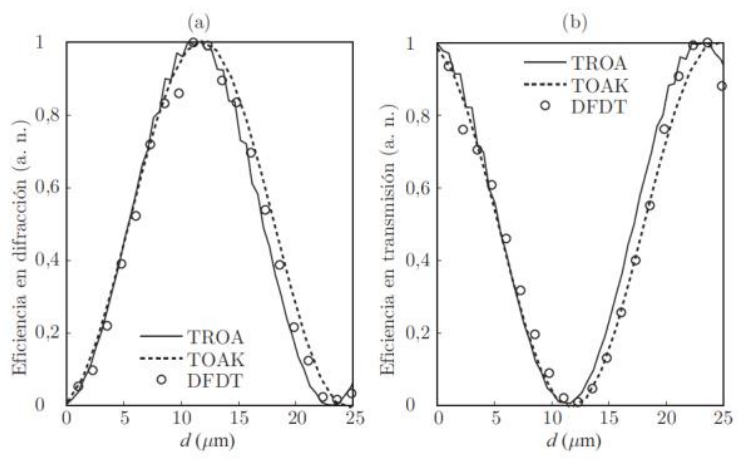

Fig. 10. Análisis del orden difractado y transmitido para una RDH en transmisión con $\Delta n=0.025$ (Francés et al. [18]). (a) Eficiencia en difracción $(m=1)$. (b) Eficiencia en transmisión $(m=0)$.

situaciones son muy comunes en programas basados en diferencias finitas [30,31], y para este caso en concreto se propuso una solución rápida y efectiva en $[16,19]$. Esta solución es ilustrada en la Fig. 9, donde al perfil sinusoidal de la red se le superpone una curva de gradiente en los bordes de la simulación para encaminar la luz generada en los extremos hacia las capas de absorción que truncan la región de simulación.

En la Fig. 10 se recogen las curvas de las eficiencia en difracción y transmisión para una red de difracción con modulación de índice $\Delta n$ de 0,025 . El período de red es de $\Lambda=0,83 \mu \mathrm{m}$ o 1200 líneas por milímetro. Se han comparado las curvas numéricas obtenidas con el método de las DFDT mediante la TROA y la teoría de Kogelnik (TOAK). La TOAK se muestra menos precisa a la hora de reproducir el comportamiento físico de estos dispositivos. Las múltiples reflexiones y los órdenes superiores rompen el perfil sinusoidal presente en la TOAK. Sin embargo, en todos los casos, el método de las DFDT proporciona unos valores satisfactorios y bastante precisos comparados con las curvas teóricas. Un análisis más amplio de la aplicación del método DFDT a este tipo de elementos difractivos se puede encontrar en $[16,18]$.

De manera similar también se ha realizado un análisis comparativo de la precisión del método de las DFDT en función de la resolución espacial. También se ha analizado la precisión del método de la matriz característica, el cual está sujeto a las mismas limitaciones en cuanto a resolución espacial se refiere. Cabe recordar que la teoría del medio efectivo (TME) se basa en aproximar mediante capas dieléctricas homogéneas el medio que se quiere estudiar [16]. Cada una de estas capas se representa mediante una matriz característica. Para el caso de las $\mathrm{RDH}$, el perfil sinusoidal de la red es muestreado y discretizado. El impacto de la discretización en la precisión de los resultados obtenidos mediante los métodos desarrollados se muestra en la Fig. 11, donde se comparan tanto el método de las DFDT, como la TME con el método basado en las funciones de Mathieu y la teoría de Kogelnik. En este caso, el método de Mathieu es la referencia teórica, ya que este método no es sensible a una discretización espacial. Un análisis extenso de esta problemática se recoge en $[16,19]$. Se puede apreciar, que en ambos casos el método DFDT se ajusta de forma satisfactoria al caso teórico (método de Mathieu), mientras que la teorías convencionales basadas en ondas acopladas se muestran inexactas en este caso concreto y particular $[16,19]$.

En lo referente a la optimización computacional, en la Fig. 12 se ilustra el rendimiento en GFlops (mil millones de operaciones en coma flotante de precisión simple por segundo) para la versión completa del método de las DFDT para dos estaciones de trabajo, así como para las dos implementaciones optimizadas en la CPU y en la GPU. En las Figs. 12(a) y 12(b) se representa el rendimiento en 
(a)

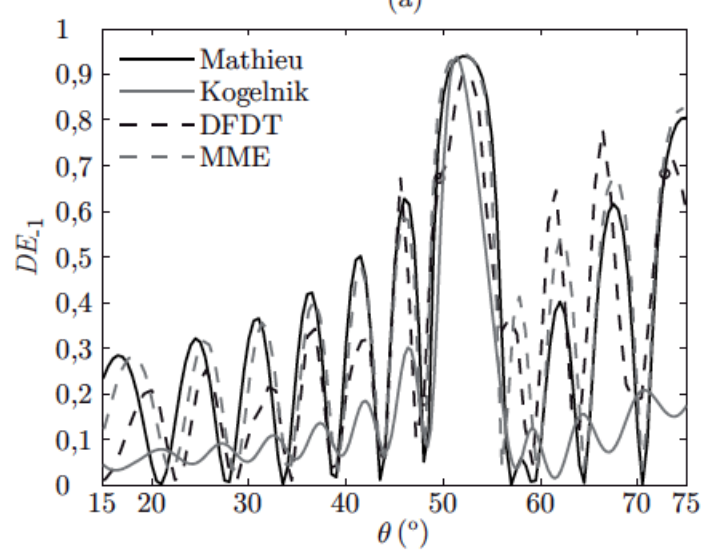

(b)

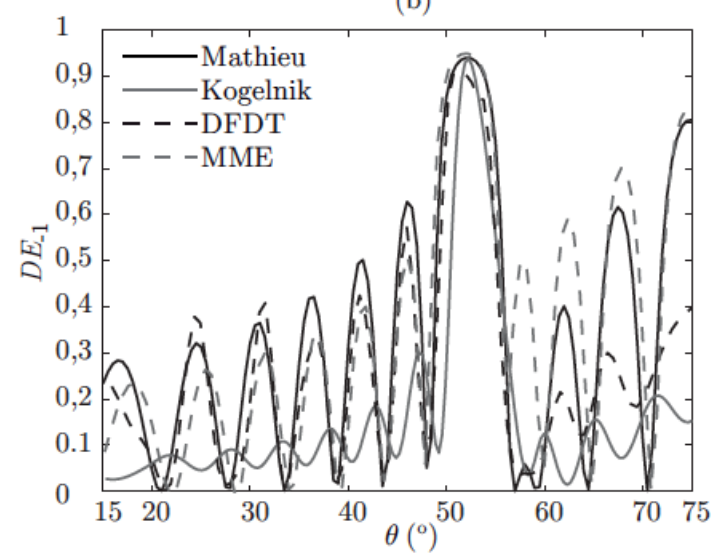

Fig. 11. Análisis de la eficiencia angular en función de la resolución espacial en los métodos de las DFDT y de la matriz efectiva. En ambas gráficas las curvas del MM y de Kogelnik son las mismas. (a) Curvas de DFDT y de MME para 8 muestras por $\Lambda$. (b) Curvas de DFDT y de MME para 16 muestras por $\Lambda[16,19]$.

(a)

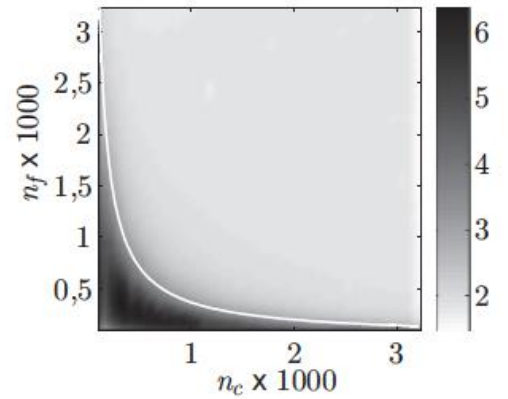

(c)

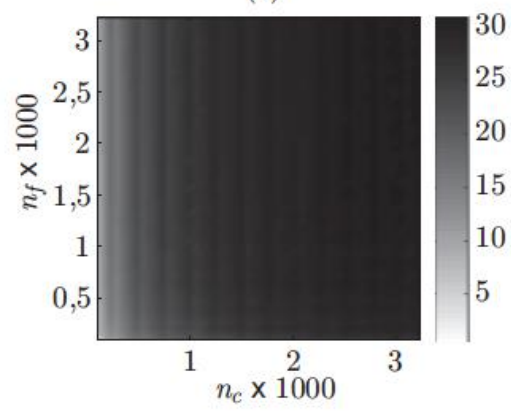

(b)

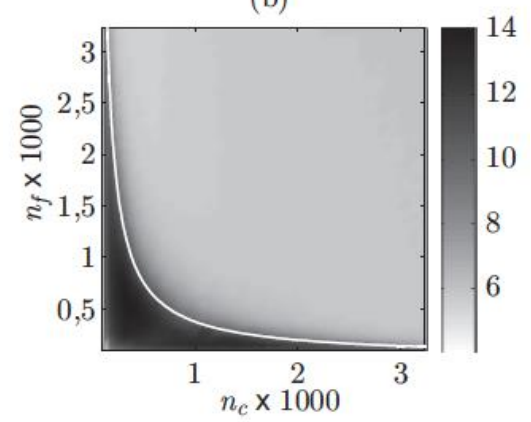

(d)

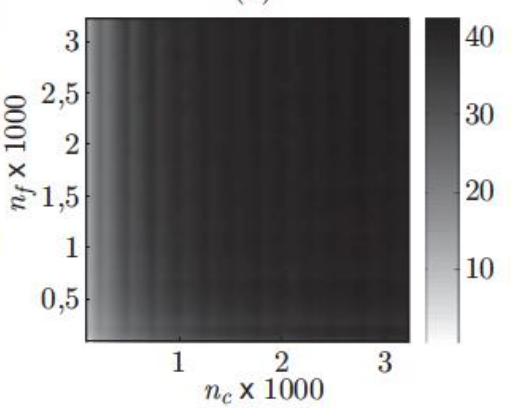

Fig. 12. Análisis de la eficiencia angular en función de la resolución espacial en los métodos de las DFDT y de la matriz efectiva. En ambas gráficas las curvas del MM y de Kogelnik son las mismas. (a) Curvas de DFDT y de MME para 8 muestras por $\Lambda$. (b) Curvas de DFDT y de MME para 16 muestras por $\Lambda[16,19]$.

función del tamaño de la simulación (definiendo la malla DFDT como una matriz de $n_{r}$ filas y $n_{c}$ columnas) para el caso de los microprocesadores Intel Quad-Core Q6600 e Intel i7-950 respectivamente. La curva blanca delimita el tamaño de la malla que excede el tamaño de caché de la CPU en una simulación completa en dos dimensiones. La zona comprendida por debajo de esta línea evidencia una región en la cual la CPU es competitiva frente a la GPU. Para el caso del microprocesador
Q6600, el rendimiento se reduce antes de lo esperado debido a la propia arquitectura del microprocesador y su menor tamaño de memoria caché. En las Figs. 12(b) y 12(c) se muestra el rendimiento para las GPU GeForce GTX-460 y 470 respectivamente. En ambas el patrón es prácticamente idéntico salvo un factor de escalado, el cual puede ser debido principalmente a la diferencia en el número de multiprocesadores (mayor en la 470), junto con la velocidad del reloj del sistema, también 
superior en la 470. Tanto para el caso de la Fig. 12 , así como el resto de análisis recogidos en $[16,20]$, muestran cotas superiores tanto en reducción de tiempos de simulación como en rendimiento, cercanos a los 40 GFlops (véase Fig. 12), estableciendo límites superiores en aplicaciones de DFDT con GPU previamente publicadas, como puede ser el trabajo realizado por Kim et al. [32].

\section{Conclusiones}

En este trabajo se han desarrollado e implementado una serie de métodos numéricos y herramientas para el análisis de elementos difractivos. Entre estos métodos se ha optimizado de forma computacional el método DFDT debido a su potencial y versatilidad de cara a ser aplicado a problemas complejos de formación de imágenes, holografía, almacenamiento y procesado óptico de la información, y diseño de lentes difractivas entre otras aplicaciones. Los resultados derivados del análisis de RDH mediante DFDT evidencian una alta precisión del método en todos los casos, al mismo tiempo que demuestra las carencias de métodos teóricos convencionales. Además, se ha desarrollado una metodología a partir de las funciones de Mathieu que establece una alternativa robusta y fiable para el análisis teórico y analítico para el caso específico de RDH con patrón de interferencia no inclinada, en las cuales la teoría basada en ondas acopladas deja de ser estrictamente aplicable. Parte del trabajo se ha aplicado a la docencia para estudiar el rango de validez de las expresiones teóricas de la difracción de aperturas en regiones de Fresnel y Fraunhofer, además de proporcionar la distribución del campo electromagnético en función del tiempo y del espacio. Finalmente se muestran los resultados derivados de la optimización del método DFDT tanto en CPU como GPU. En concreto, la versión en GPU se compara de forma rigurosa con una versión optimizada en CPU que utiliza instrucciones vectoriales y distribuye la carga de cálculo en diversos hilos de proceso. Los resultados corroboran que las GPU se muestran más competitivas para simulaciones de alto coste temporal.

\section{Agradecimientos}

El autor desea agradecer la inestimable ayuda y apoyo de sus directores de tesis Cristian Neipp y Andrés Márquez. También destacar a diversas personas que también han contribuido en la realización de este trabajo dentro del GHPO de la UA: Sergio Bleda, Sergi Gallego, Manuel Ortuño, Augusto Beléndez e Inmaculada Pascual.

Este trabajo ha sido parcialmente financiado por el Ministerio de Economía y Competitividad de España a través del proyectos FIS201129803-C02-01, por la Universidad de Alicante a través del proyecto GRE12-14 y por la Generalitat Valenciana a través del proyecto GV/2014/076. 